
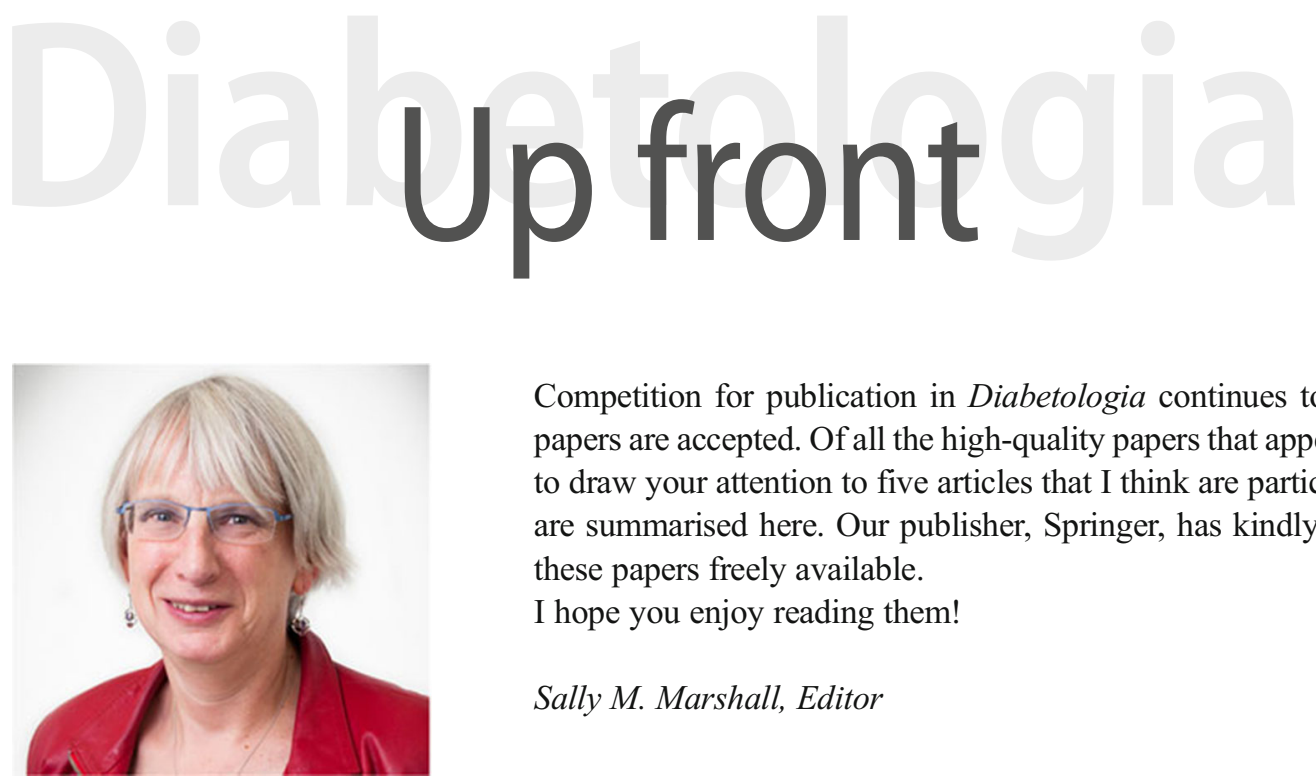

Competition for publication in Diabetologia continues to grow, and less than $20 \%$ of papers are accepted. Of all the high-quality papers that appear in this month's issue I want to draw your attention to five articles that I think are particularly interesting. The articles are summarised here. Our publisher, Springer, has kindly made the full text of each of these papers freely available.

I hope you enjoy reading them!

Sally M. Marshall, Editor

2019 update to: Management of hyperglycaemia in type 2 diabetes, 2018. A consensus report by the American Diabetes Association (ADA) and the European Association for the Study of Diabetes (EASD)

John B. Buse, Deborah J. Wexler, Apostolos Tsapas, Peter Rossing, Geltrude Mingrone, Chantal Mathieu, David A. D’Alessio, Melanie J. Davies

In 2018, the ADA/EASD published a consensus report for the management of hyperglycaemia in type 2 diabetes (https://link. springer.com/article/10.1007/s00125-018-4729-5). Patientcentred care that was focused on reducing the burden of complications represented a paradigm shift in the approach to diabetes management, with the preferred use of glucagon-like peptide 1 receptor agonists (GLP-1 RAs) and sodium-glucose cotransporter 2 (SGLT2) inhibitors in those with established atherosclerotic cardiovascular disease (ASCVD). Since then, there has been an unprecedented release of new outcome data, mandating a brief update to the 2018 consensus. In this issue, the ADA/EASD (https://doi.org/10.1007/s00125-019-05039w) present important changes to the 2018 consensus, including consideration of GLP-1 RAs and SGLT2 inhibitors for beneficial cardiorenal outcomes in individuals with type 2 diabetes, independently of $\mathrm{HbA}_{1 \mathrm{c}}$ levels. Where major adverse cardiovascular events are the gravest threat, the evidence favours the preferred use of GLP-1 RAs; as well as being used in those with type 2 diabetes and established ASCVD, their use should be considered in those with specific indicators of high cardiovascular risk. When it comes to the use of SGLT-2 inhibitors in type 2 diabetes, the evidence is now much clearer for their benefit, particularly in those with heart failure with reduced ejection fraction and in those with chronic kidney disease (CKD), and in the prevention of CKD progression. Thus, SGLT-2 inhibitors are preferred for diabetes management in individuals with these comorbidities.

\section{Positioning time in range in diabetes management}

Andrew Advani

The time in range (TIR) metric has emerged as being a key indicator of the quality of glucose control in diabetes. In this issue, Andrew Advani (https://doi.org/10.1007/ s00125-019-05027-0) summarises recent TIR research with the aim of assisting healthcare professionals and individuals living with diabetes in interpreting TIR data and setting TIR goals. Evidence is beginning to emerge linking reduced TIR with increased risk of long-term diabetes complications and adverse pregnancy outcomes. This association may be expected given the inverse correlation between TIR and $\mathrm{HbA}_{1 \mathrm{c}}$ levels. The author explains that, because of the skewed distribution of glucose levels outside the target range, TIR (on its own) is a poor indicator of the frequency and severity of hypoglycaemia. Thus, all TIR discussions should separately consider time below range and, by inference, time above range. In this review, the recent consensus recommendations for time in ranges goals are summarised, although, as is always the case, glycaemic targets should be individualised. (b) The figures from this review are available as a downloadable slideset. 
Non-alcoholic fatty liver disease and cardiovascular disease: assessing the evidence for causality

Martijn C. G. J. Brouwers, Nynke Simons, Coen D. A. Stehouwer, Aaron Isaacs

Non-alcoholic fatty liver disease (NAFLD) has emerged as a risk factor for type 2 diabetes and cardiovascular disease (CVD). In this issue, Brouwers and colleagues (https://doi. org/10.1007/s00125-019-05024-3) elaborate on potential mediators of the relationship between NAFLD and CVD, such as plasma lipids, low-grade inflammation, impaired fibrinolysis and hepatokines. By summarising recent advances in genetic studies that focused on the relationship between NAFLD susceptibility genes (PNPLA3, TM6SF2, GCKR and $M B O A T 7$ ) and CVD, it appears that plasma lipids are an important mediator of that relationship. This has therapeutic implications, particularly for the design of anti-NAFLD drugs that affect lipid metabolism. Since these agents are aimed primarily at preventing end-stage liver disease, it is important to underscore the fact that the principal cause of death in individuals with NAFLD is CVD.

(5) The figures from this review are available as a downloadable slideset.

Stearoyl CoA desaturase is a gatekeeper that protects human beta cells against lipotoxicity and maintains their identity

Masaya Oshima, Séverine Pechberty, Lara Bellini, Sven O. Göpel, Mélanie Campana, Claude Rouch, Julien Dairou, Cristina Cosentino, Federica Fantuzzi, Sanna Toivonen, Piero Marchetti, Christophe Magnan, Miriam Cnop, Hervé Le Stunff, Raphaël Scharfmann

Rodent beta cells are highly sensitive to treatment with saturated NEFA, leading to their impairment and death. However, translating data from rodent to human beta cells remains challenging. Previous data indicate that treatment of a recently engineered functional beta cell line, EndoC- $\beta \mathrm{H} 1$, with palmitate does not induce lipotoxicity under standard culture condi- tions. In this issue, Oshima et al (https://doi.org/10.1007/ s00125-019-05046-x) report that, upon stearoyl CoA desaturase (SCD) knockdown, EndoC- $\beta \mathrm{H} 1$ cells are sensitive to lipotoxicity and that they dedifferentiate. Treatment with long chain saturated NEFA exacerbates dedifferentiation and induces markers of inflammation and endoplasmic reticulum stress. The authors suggest that characterising the factors that govern SCD activity may lead to new approaches to overcome pancreatic beta cell dedifferentiation, dysfunction and apoptosis during type 2 diabetes.

An artificial intelligence-based deep learning algorithm for the diagnosis of diabetic neuropathy using corneal confocal microscopy: a development and validation study

Bryan M. Williams, Davide Borroni, Rongjun Liu, Yitian Zhao, Jiong Zhang, Jonathan Lim, Baikai Ma, Vito Romano, Hong Qi, Maryam Ferdousi, Ioannis N. Petropoulos, Georgios Ponirakis, Stephen Kaye, Rayaz A. Malik, Uazman Alam, Yalin Zheng

Diabetic neuropathy is the strongest initiating risk factor for foot ulceration and amputations. Current 'screening' practices are highly subjective and only diagnose diabetic neuropathy when well-established, which is in stark contrast to the tools available for the early diagnosis of other diabetic complications, such as retinopathy. Future development of valid screening programmes utilising technology that detects early neuropathy is, therefore, of paramount importance and clinical need. In this issue, Williams et al (https://doi.org/10.1007/s00125-019-05023-4) present an artificial intelligence-based algorithm that accurately and rapidly detects diabetic neuropathy through noninvasive assessment of the corneal sub-basal nerve plexus using corneal confocal microscopy. They show that this approach outperforms the currently used automated software, with superior intraclass correlation coefficients for all major corneal nerve biomarkers. The authors conclude that these findings indicate that corneal confocal micros copy is now primed for population-based screening of diabetic neuropathy.

All text supplied by the authors. 